\title{
Ice crystallization observed in highly supercooled confined water ${ }^{\dagger}$
}

\author{
E. Stefanutti, ${ }^{a}$ L. E. Bove, ${ }^{b \neq}$ G. Lelong, ${ }^{b}$ M. A. Ricci, ${ }^{a}$ A. K. Soper, ${ }^{c}$ and F. Bruni ${ }^{* a}$
}

We investigate the state of water confined in the cylindrical pores of MCM-41 type mesoporous silica, with pore diameters of $2.8 \mathrm{~nm}$ and $4.5 \mathrm{~nm}$, over the temperature range $160-290 \mathrm{~K}$ by combining small angle neutron scattering and wide angle diffraction. This allows us to observe simultaneously the intermolecular correlations in the local water structure (which shows up in a main water peak around $Q=1.7 \AA^{-1}$ ), the two-dimensional hexagonal arrangement of water cylinders in the silica matrix (which gives rise to a pronounced Bragg peak around $Q=0.2 \AA^{-1}$ ), and the so-called Porod scattering at smaller $Q$, which arises from larger scale interfacial scattering within the material. In the literature, the temperature evolution of the intensity of this Bragg peak has been interpreted as the signature of a density minimum in confined water at approximately $210 \mathrm{~K}$. Here we show that, under the conditions of our experiment, a fraction of freezable water coexists with a layer of non-freezable water within the pore volume. The temperature dependence of all three aspects of our data, as well as comparing the data from two different pore sizes, leads us to conclude that the observed variation in the intensity of the main Bragg diffraction peak is actually caused by a liquid to ice transition in the freezable fraction of confined water.

\section{Introduction}

The density maximum of liquid water at $277 \mathrm{~K}^{1}$ is one of the best known water anomalies as it has a direct impact on our daily life and on our ecosystem. Water, indeed, as few other anomalous liquids, displays a maximum density in the liquid phase, related to a negative thermal expansion coefficient, and implying that the liquid expands upon cooling or shrinks upon heating. As an example, this anomalous expansion plays an important role by only freezing the upper layer in lakes and rivers, leaving life in the bottom layers free to develop. Hydrogen bonding between water molecules and their local tetrahedral arrangement, which characterizes both the liquid and the several crystalline ice phases, are responsible at a microscopic level for this anomaly. The presence of a density maximum suggests the possible existence of a

\footnotetext{
${ }^{a}$ Dipartimento di Scienze, Università degli Studi Roma Tre, Via della Vasca Navale 84, 00146 Roma, Italy

${ }^{b}$ Sorbonne Université, Muséum National d?Histoire Naturelle, UMR CNRS 7590, IRD, Institut de Minéralogie, de Physique des Matériaux et de Cosmochimie, IMPMC, 4 Place Jussieu, 75005 Paris, France

${ }^{c}$ ISIS Department, UKRI-STFC Rutherford Appleton Laboratory, Harwell Campus, Didcot, OX11 OQX, U.K.

*; E-mail: fabio.bruni@uniroma3.it

$\dagger$ Electronic Supplementary Information (ESI) available: [details of any supplementary information available should be included here]. See DOI: 10.1039/cXCP00000x/

$\ddagger$ Present address: Dipartimento di Fisica, Sapienza Università di Roma, P-le A. Moro 2, 00185 Roma, Italy
}

density minimum in the supercooled region ${ }^{2}$, because the density of water decreases rapidly below the temperature of the density maximum and its value can not go below that of ice.

The quest for this density minimum ${ }^{3-5}$, as well as for the observed thermodynamic anomalies in the supercooled region, has prompted the formulation of different hypotheses to describe undercooled water. Among other proposals, of particular interest is the idea that, at low enough temperatures, there exist two metastable liquid phases, namely low-density (LDL) and high density (HDL) water, whose domain of existence is believed to be separated by a first-order transition line ending in a second critical point ${ }^{5}$.

The hypothesis for a liquid-liquid transition in supercooled water has been tested in a number of computer simulation studies. Both the predicted critical temperature and pressure vary quite widely between the different models ${ }^{6-10}$. Mean field models which have a second critical point have also been quite successful at predicting thermodynamic properties of water, but give a different prediction for the location of the second critical point compared to the computer simulation results ${ }^{11,12}$. The mW model of water is particularly interesting in this regard, since it predicts quite successfully several water anomalies, including a density maximum at $T>0$ and a density minimum in the supercooled region, but at low enough temperature the ice nucleation time becomes faster than the liquid relaxation time, therefore no liquid-liquid transition is observed ${ }^{13,14}$. Under the assumption 
that there is a second critical point in water, the extension of the phase boundary into the single phase region of the $P-T$ plane, the so-called Widom line ${ }^{15}$, typically intersects the temperature axis at around 210 - $230 \mathrm{~K}$ at ambient pressure. Within this scenario, this latter temperature should be close to that of the water density minimum.

Experimental evidence for the presence of a density minimum is hard to obtain. This is because, when cooling the supercooled liquid, the onset of homogeneous crystal nucleation occurs at a temperature, $\mathrm{T}_{\mathrm{H}}, \sim 235 \mathrm{~K}$. Attempts to explore the supercooled region from below, i.e. by melting the amorphous phases, are hindered by the spontaneous crystallization of ice at $\sim 150 \mathrm{~K}$. These two temperatures delimit the so-called no man's land, a region of the phase diagram where probing bulk liquid water appears impossible. However recent X-ray laser experiments appear to have penetrated a few degrees K into no-man's land for water ${ }^{16,17}$. Using femto-second X-ray pulses and the huge intensity available at such facilities, it is possible to capture the structure of individual micron-sized droplets immediately before they crystallize below $\mathrm{T}_{\mathrm{H}}$. The authors of that work claim they have seen a peak in the isothermal compressibility, at $\sim 229 \mathrm{~K}$, which, if it were true, could correspond to the crossing of the purported Widom line, although other explanations of the data cannot be discounted ${ }^{18}$.

In the past decade or so, in order to circumvent such hurdles, a series of experiments have been performed on water confined in nanoporous materials. Upon confinement, the melting temperature of water is lowered and apparently crystallization is only observed, by means of Differential Scanning Calorimetry (DSC), for pore diameters larger than $\sim 3.0 \mathrm{~nm}^{19,20}$. For water confined in smaller pores the suppression of crystallization would, allow confined water to remain in the deeply supercooled state below the homogeneous crystallization temperature of bulk water. This approach has been used to derive hints of the possible existence of a liquid-liquid phase transition in the no man's land of bulk water, provided that confined water can be assumed to be a realistic model for bulk water ${ }^{21,22}$. Whatever the nature of the surface of the porous matrix (polar, hydrophilic or hydrophobic), the restriction of the accessible space induced by confinement has indeed important structural, dynamical and thermodynamical consequences. Despite many experimental and computational studies, the effects of a specific nanoconfinement on the properties of water are still largely unknown.

The first experimental claim for evidence of a density minimum in confined water was obtained with small angle neutron scattering (SANS) on water confined in the cylindrical pores of MCM-41$\mathrm{S}-15$ with nominal pore diameter of $1.5 \pm 0.1 \mathrm{~nm}$, arranged in a $2 \mathrm{D}$ hexagonal lattice ${ }^{24}$. The rationale behind this experiment was to consider the intensity of the (10) Bragg peak, which arises from the $2 \mathrm{D}$ hexagonal array of unidirectional pores, as proportional to the difference between the scattering density of the confined liquid and that of the confining substrate, under the assumption of a uniform water density distribution across the diameter of the pore. This assumption plays a crucial role in the interpretation of the data, as we shall see in the following.

This first study was followed by other experiments and simulations, which either confirmed the finding or criticized it. Briefly, supporting evidence has been provided by similar small angle neutron or X-ray scattering (SAXS) studies ${ }^{25-27}$. In addition, crystallization at $220 \mathrm{~K}$ has been observed for water confined in MCM41 with larger pore size $(2.4 \mathrm{~nm})^{27}$. The apparent lack of a liquid water density minimum in this latter case was considered as an overall validation of the DSC data and of the method based on the intensity of the (10) Bragg peak as a probe of water density under confinement. An overview of these findings, discussing both the density minimum and the hypothesized liquid-liquid critical point, can be found in reference ${ }^{28}$. Conversely, atomistic simulations of wide angle scattering data on water confined in MCM material with nominal pore diameter of $1.5 \pm 0.1 \mathrm{~nm}$ pointed out that the water density profile across the pore is not flat and can change with temperature ${ }^{29}$. Other simulations of similar systems also reported a non-uniform density profile, which included the idea that there is a layer of "bound" water near the pore surface different from bulk water ${ }^{30,31}$. It must be also recalled that a temperature dependent density, in turn, would lead to a change in Bragg peak intensity, even when the overall density across the pore stays constant ${ }^{23,32}$. SAXS experiments on water confined in MCM-41-S material with pore size ranging from 2.0 to $4.4 \mathrm{~nm}$ were used to demonstrate that the scattering data cannot be described as resulting from a homogeneous density distribution of water within the pores ${ }^{33}$. Interestingly, in the same study ${ }^{33}$, the authors reported the presence of a water fraction in the center of the pore with different properties compared to that at the pore wall, and identified a density minimum similar to that found by Liu et al. ${ }^{24}$. However, at odds with ${ }^{27}$, density minima were apparently observed ${ }^{33}$ for all investigated samples, even when water was confined in MCM with the largest pore size.

NMR studies revealed that confined liquid water exhibits a high-temperature crossover of rotational motion at 220âLŠ $230 \mathrm{~K}$, which coincides with an emergence of solid water, where, most probably, the liquid and solid fractions can be identified with interfacial and internal waters, respectively ${ }^{34,35}$.

Notably, the temperature of the density minimum was found to depend on the pore size, shifting to lower temperatures as the pore diameter decreases ${ }^{33}$. This last result casts some doubt on the use of confined water as a tool to probe the existence of a density minimum in bulk water, because such a minimum should be an intrinsic characteristic of water, not depending on the size of the confining matrix. Very recently, in a series of measurements on the velocity of sound in stretched water (negative pressure) in micron-sized occlusions, a line of compressibility maxima (corresponding approximately to the Widom line) has been identified which proceeds to lower temperature as the pressure is raised ${ }^{36}$. The temperature at which this line would cross the ambient pressure line is unclear at this stage, but, assuming it does continue into the positive pressure region of the water phase diagram, its observation is at least consistent with the liquid-liquid transition hypothesis, though it does not rule out other interpretations either. Hence, as it currently stands, it would be fair to state that the question of the presence of a density minimum in deeply supercooled water and the associated crossing of the Widom line, along with a liquid-liquid transition at lower temperatures, remains unresolved. 
The aim of the present study is to shed light on the above issues, exploiting the possibility offered by the NIMROD diffractometer $^{37}$, at the ISIS pulsed neutron source, to investigate a wide $Q$ range, extending from $10^{-2}$ to $30 \AA^{-1}$, thus combining traditional SANS and wide angle diffraction in a single experiment. This is of particular importance here, because it means it is possible to look at the peak (around $Q=0.2 \AA^{-1}$ ) resulting from the (10) plane of the 2D hexagonal arrangement of the water cylinders in the silica matrix and at the main water peak (around $Q=1.7 \AA^{-1}$ ) at the same time.

As a consequence, we can, simultaneously, tackle the question of the purported density minimum, check the phase of the confined water, and observe the scattering in the very low $Q$ region. This latter $Q$ region provides information on the density fluctuations, that are expected when approaching the liquid-liquid transition temperature.

\section{Materials and Methods}

We report neutron diffraction (ND) experiments performed on water confined in MCM-41/C10 (pore size $2.8 \mathrm{~nm}$ ) and in MCM$41 / \mathrm{C} 18$ (pore size $4.5 \mathrm{~nm}$ ) as a function of temperature, in the range 160 - $290 \mathrm{~K}$. Details on the synthesis procedure are described elsewhere ${ }^{38}$. The smallest pore size was chosen because no water freezing has been observed with differential scanning calorimetry (DSC) in MCM-41 with pore diameter $\leq 3 \mathrm{~nm}^{39,40}$. To better exploit NIMROD potentialities and to distinguish features of the dry MCM material from those due to confined water, three samples were investigated for each MCM, namely a dry MCM matrix, one filled with $\mathrm{D}_{2} \mathrm{O}$, and one filled with a "zero-water" mixture made up of $92 \% \mathrm{H}_{2} \mathrm{O}$ and $8 \% \mathrm{D}_{2} \mathrm{O}$. The neutron scattering length of this mixture is such that the average coherent scattering length of the water molecule as a whole is zero, so that in the event that the water is randomly distributed and orientated across the pore there should be no coherent water scattering, which in turn would mean the scattering signal from this sample should be the same as the dry MCM-41. MCM samples were carefully hydrated up to $90 \%$ of the total pore volume available, to rule out the possibility of observing contributions to the scattering pattern from water molecules that left the pore volume due to expansion on cooling, either due to the presence of a density minimum or due to crystallization.

\section{Experimental Data and Discussion}

Figure 1 shows the raw scattering data collected at $T=284 \mathrm{~K}$ for the MCM-41/C10 samples (similar data have been collected for the larger pore size MCM-41/C18). This figure illustrates several interesting features. First, the steep rise at very low $Q$ values due to interfacial (Porod) scattering. Second, the peak around $Q=0.2^{-1}$, due to the (10) plane of the 2D hexagonal arrangement of water cylinders in the silica matrix. Third, the water peak around $Q=1.7^{-1}$, which is better visible in the figure insert. Also to be noted is that the zero-water sample is not identical to the dry MCM signal, suggesting a non-uniform distribution of water across the pore. Finally, we note that, at this temperature, the main water diffraction peak is at a markedly lower $Q$ value than is found in bulk water, suggesting that the confined water is on average at lower density than bulk water.

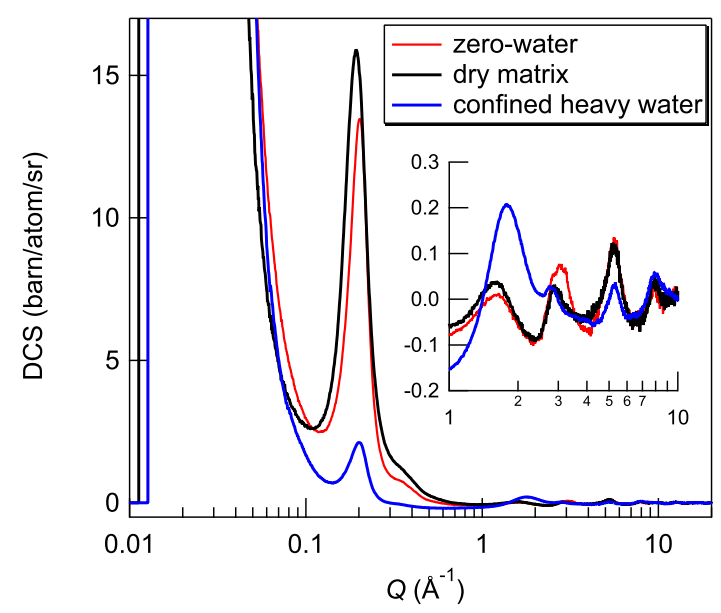

Fig. 1 Color On-line. Raw Differential Cross Section (DCS) data of MCM$41 / \mathrm{C} 10$ sample (pore size $2.8 \mathrm{~nm}$ ), hydrated with heavy water $\left(D_{2} O\right)$ or with the zero-water mixture $\left(92 \% \mathrm{H}_{2} \mathrm{O}\right.$ and $\left.8 \% \mathrm{D}_{2} \mathrm{O}\right)$ compared to data obtained for the dry matrix. The zero-water mixture composition was specifically calculated to suppress the signal from water inside the pores. The peak around $Q=0.2 \AA^{-1}$ is due to the (10) plane of the $2 \mathrm{D}$ hexagonal arrangement of water cylinders in the silica matrix. The peak around $Q=1.7 \AA^{-1}$, better visible in the figure insert, is ascribed to water.

Following the same procedure described in ${ }^{24}$, we investigated the temperature dependence of the intensity of the Bragg peak around $Q=0.2 \AA^{-1}$, treating it, as in ${ }^{24}$, as a direct measure of the average mass density of water in the pores.

The first step of the procedure requires a subtraction of a power law decay from each diffraction pattern, in order to remove the contribution from interfacial scattering. Details on this procedure and on the data analysis methods adopted to investigate the temperature dependence of the Bragg peak are described in the Supplementary Information.

Figure 2 shows the temperature dependence of the Bragg peak after background subtraction, along with the fits obtained with eq. 4 in the Supplementary Information. We remark the very good agreement between the model and the experimental data. The mass density of $\mathrm{D}_{2} \mathrm{O}$, extracted as a fit parameter, is plotted as a function of temperature in Figure $3 \mathrm{~A}$, and compared to the trend reported in ${ }^{24}$. Even though the absolute values of the mass density of confined $\mathrm{D}_{2} \mathrm{O}$ may not be reliable, yet the adopted normalization does not affect its temperature dependence.

The measured apparent $\mathrm{D}_{2} \mathrm{O}$ density shows a minimum at about $210 \mathrm{~K}$, albeit less marked compared to previous data ${ }^{24,41}$. However, it should be noted that the accuracy of the density values extracted from the fitting procedure strongly depends on the background subtraction. Figure 3B shows the temperature dependence of the thermal expansion coefficient $\alpha_{P}=-\rho_{m}^{-1}\left(\partial \rho_{m} / \partial T\right)_{P}$, calculated from the data in Fig. 3A. If we compare our results (red markers) with those obtained in ${ }^{24}$ (black markers), we can notice a good overall agreement. The temperature of the density minimum corresponds to the temperature where $\alpha_{P}$ is equal to zero, and $\alpha_{P}$ is expected to exhibit a maximum crossing the Widom line 


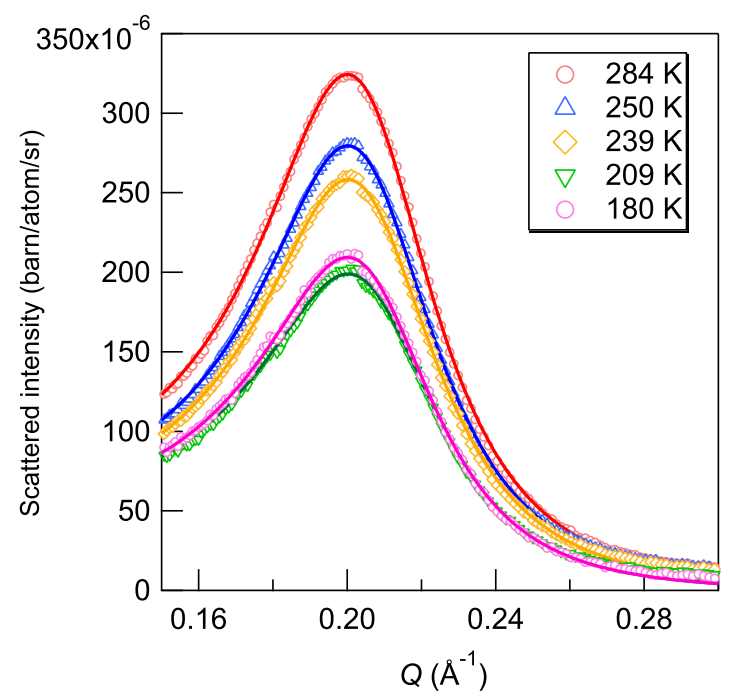

Fig. 2 Color On-line. Analysis of the Bragg peak intensity with the model given by eq. 4 in the Supplementary Information. Experimental neutron scattering intensity (open symbols) obtained with the $\mathrm{D}_{2} \mathrm{O}$-hydrated MCM-41/C10 sample and fits (solid lines) according to eq. 4 in the Supplementary Information. To make the figure clearer, only some selected temperatures have been shown. However, the same good agreement between the model and the experimental data is observed at all the investigated temperatures.

above the critical point ${ }^{27,42}$; this implies that both data sets suggest a density minimum in the temperature range $210-215 \mathrm{~K}$, and a crossing of the Widom line around $240 \mathrm{~K}$ at ambient pressure.

As discussed above, if below a second critical point supercooled water is regarded as a mixture of two states of the same liquid, differing in density $3,28,43-47$, then density fluctuations above that critical point will reach a maximum close to the Widom line. In principle, this should, in turn, give rise to enhanced scattering intensity at low $Q$ near the transition temperature ${ }^{48}$. In order to make this point clearer, we have performed a quantitative, model free, analysis of the Porod region, looking at the scattered intensity at selected $Q$ values as a function of temperature. The results (Fig. 4) show a continuous decrease of the scattered intensity with increasing $Q$. For $Q$ values below $0.059 \AA^{-1}$ the scattered intensity shows no (or very weak) temperature dependence, suggesting the absence of density fluctuations and, in turn, of two coexisting liquid states. Such a result is remarkable as it clearly provides evidence that the Widom line is not crossed upon cooling confined $\mathrm{D}_{2} \mathrm{O}$ at ambient pressure. This result is at odds with a recent report, based on extremely fast X-ray diffraction in supercooled micron-sized water droplets ${ }^{17}$. However, even in this latter work the rise in scattering at low $Q$ is very modest in practice. Interestingly, the drop in intensity with temperature (below $T=240 \mathrm{~K}$ and for $Q \geq 0.059 \AA^{-1}$ ) indicates a reduction in compressibility, as it would be expected for a solidification transition.

At this stage, we appear to have two conflicting pieces of evidence: an apparent density minimum at about $210 \mathrm{~K}$, along with an associated maximum in the thermal expansion coefficient at about $240 \mathrm{~K}$, on the one hand, and the absence of obviously enhanced density fluctuations at low $Q$ at all investigated tempera-
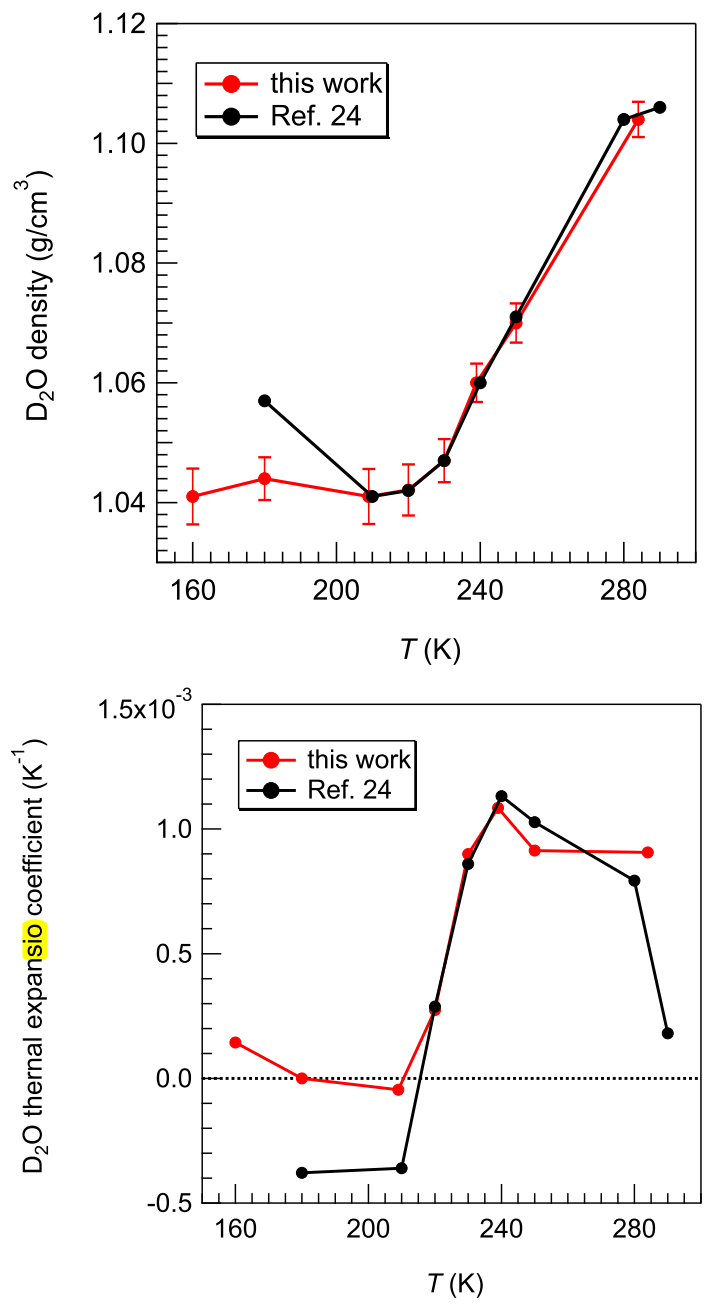

Fig. 3 Color On-line. (A) Comparison between the temperature dependence of the mass density of $\mathrm{D}_{2} \mathrm{O}$ calculated in the present work (red symbols) with those reported in ${ }^{24}$ (black symbols). (B) Thermal expansion coefficient, $\alpha_{P}=-\rho_{m}^{-1}\left(\partial \rho_{m} / \partial T\right)_{P}$, as a function of temperature. $\alpha_{P}$ has been calculated with the $\rho_{m}$ data shown on Fig. 3A; those indicated with red symbols have been obtained from our experimental data, while those indicated with black symbols have been calculated using data reported in ${ }^{24}$. Lines connecting symbols are guides for eyes.

tures on the other. To reconcile these two observations, we looked at the short-range correlation of confined $\mathrm{D}_{2} \mathrm{O}$. In particular, we investigated in detail the temperature dependence of the main water peak around $Q=1.7 \AA^{-1}$ (see Fig. 1, Fig.1 insert, and Fig. 5 ), the rationale being that any modifications of the diffraction profile must come from changes in the state of confined water ${ }^{52}$.

Figure 5 shows the scattering data in two $Q$ regions, namely in the low $Q$ region of the (10) Bragg peak, and in the region of the main water diffraction peak $\left(1 \leq Q \leq 6 \AA^{-1}\right.$, right hand graphs), for both MCM-41/C10 (top row) and MCM-41/C18 (bottom row). Starting from the left, plot (A), we notice that the temperature dependence of the (10) Bragg peak in the $2.8 \mathrm{~nm}$ pores of MCM-41/C10, already discussed above and shown in detail in Figure 2, goes along with a change in position and reduction in width of the main water peak on cooling below $240 \mathrm{~K}$, as shown in plot (B). The observation of a peak narrowing strongly sug- 


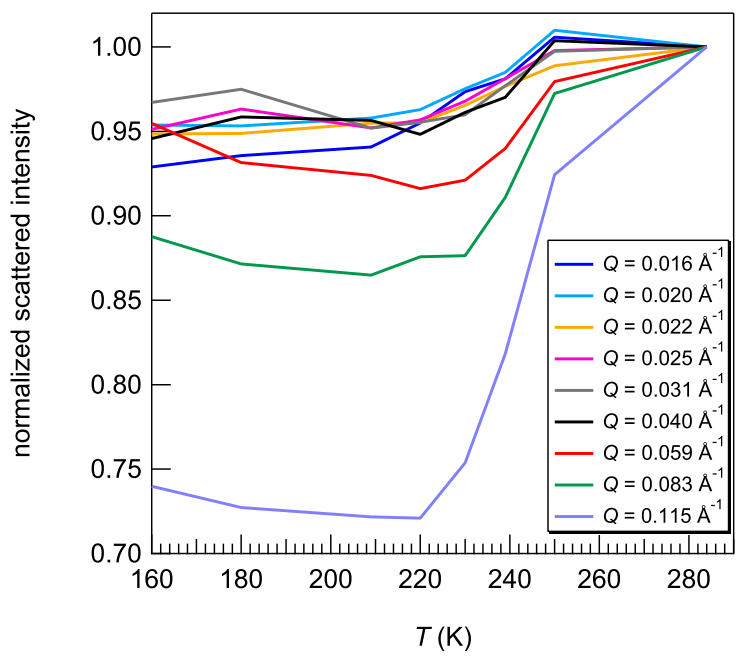

Fig. 4 Color On-line. Temperature dependence of the Porod region of DCS obtained with $\mathrm{D}_{2} \mathrm{O}$ confined in MCM-41/C10 sample at several $Q$ values. For each $Q$ value the DCS data have been normalized to their value at $T=280 \mathrm{~K}$. The figure shows that below $Q=0.59 \AA^{-1}$ the scattered intensity does not depend strongly on temperature.

gests crystallization or solidification of at least a fraction of confined water (more on this issue below). At temperatures below $210 \mathrm{~K}$ further changes are relatively small. The situation is similar for the larger pores of MCM-41/C18, plots (C) and (D). Also in this case, the temperature dependent decrease of intensity of the Bragg peak is accompanied by a sharp change in the position and width of the main water diffraction peak between $T=240 \mathrm{~K}$ and $T=230 \mathrm{~K}$, with minor changes below $230 \mathrm{~K}$. Once the substantial background from Porod scattering at very low $Q$ has been removed (see Supplementary Information for a detailed description of this procedure), for both MCM materials the intensity of the (10) peak drops as the temperature is lowered: by a factor of $\sim 50 \%$ for $2.8 \mathrm{~nm}$ pores, and $\sim 33 \%$ for $4.5 \mathrm{~nm}$ pores. The drop in intensity levels out at the temperature where crystallization or solidification reaches completion in both cases $(230 \mathrm{~K}$ for $4.5 \mathrm{~nm}$ pores, $210 \mathrm{~K}$ for $2.8 \mathrm{~nm}$ pores); notably this is the temperature at which a density minimum in the confined water has been reported.

The comparison between MCM-41/C10 and MCM-41/C18 regarding the temperature evolution of the water main diffraction peak, points to a more sudden change with temperature and a sharper main peak at the lowest temperature for water confined in MCM-41/C18. These observations can be accounted for by considering the larger constraints imposed by confinement of water in MCM-41/C10 and the substantial pore size broadening in this latter case. However, the narrowing and shift of the main peak is unequivocal for both pore sizes.

Remarkably, the main diffraction peak of water confined in MCM-41/C10 no longer appears as a single peak below $240 \mathrm{~K}$, but rather looks like the convolution of substructures, located approximately between 1.5 and $1.9 \AA^{-1}$. These substructures were not present in the liquid phase, and can be assigned to the formation of stacking faults ice ${ }^{49}$ albeit with a change in intensities due to the likely partially oriented nature of the growing crystallites.
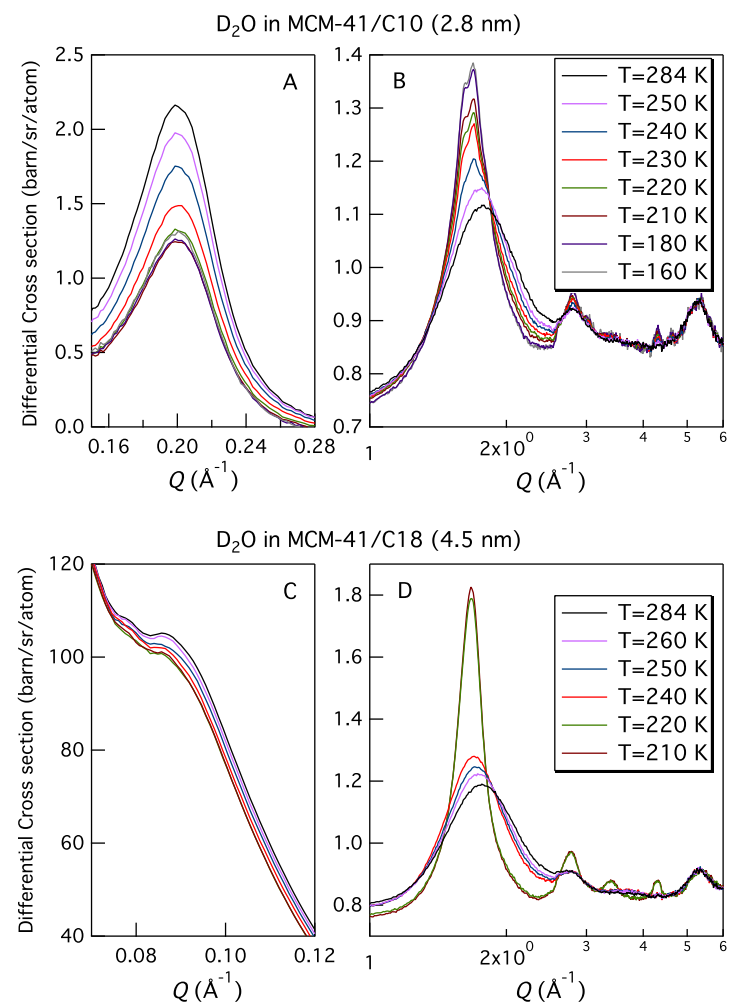

Fig. 5 Color On-line. Comparison between the differential cross sections of $\mathrm{D}_{2} \mathrm{O}$ in MCM-41/C 10 and MCM-41/C18 in the low $Q$-region, plots (A) and $(\mathrm{C})$, and in the range of the main water diffraction peak, $1 \leq Q \leq 6$ $\AA^{-1}$, plots (B) and (D). Details on the procedure adopted to remove the substantial background from Porod scattering at very low $Q$, particularly evident in plot $(\mathrm{C})$, are given in the Supplementary Information.

The observation of stacking faults ice $I$ formation when water crystallizes at low temperature is a well known phenomenon observed when water freezes from vapor deposited nanodroplets ${ }^{50}$, or from salty solutions ${ }^{51}$, as well as in other environments favoring deep undercooling ${ }^{49}$. Interestingly, the intensity of these small features does not appear to grow as the temperature is lowered, suggesting they do not arise from water extruding from the pores as proposed by ${ }^{26}$, but may arise from a small amount of water in larger interstices in the MCM structure. However, the assignment of the freezable fraction of water to a homogeneous ice structure is not straightforward.

We report in Figure 6 a comparison of a diffractogram of stacking faults ice $I$, as shown in Figure 5 of ref. ${ }^{49}$, and the diffraction pattern of $\mathrm{D}_{2} \mathrm{O}$ confined in MCM-41/C10 and MCM-41/C18 at $209 \mathrm{~K}$. We can easily recognize stacking disordered ice $I$ main peaks at $1.6,1.7,2.8$ and $4.3 \AA^{-1}$. The lack of some - minor - reflections in the black curve in Fig. 6 (D2O in MCM-41/C10) with respect to the reference diffractogram can be attributed to the non perfectly powdered nature of the ice growing in the nanopores. Moreover, stacking sequences can be significantly different depending on the thermal history of the ice sample. The comparison here with stacking faults ice $I$ obtained by crystallization of recovered ice $\mathrm{V}$ is therefore only indicative. The difference in stacking sequences (see ref. ${ }^{49}$ ) can also explain why ice $I$ structure ob- 


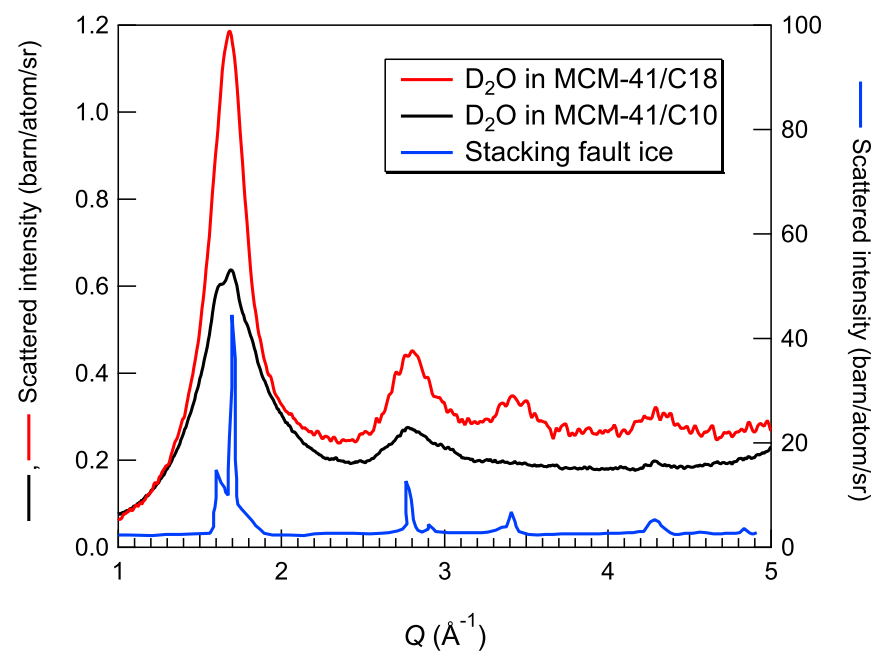

Fig. 6 Color On-line. Comparison between the diffractogram of stacking fault ice $I$ (data taken from ref. ${ }^{49}$, blue line) with the experimental differential cross-section of $D_{2} \mathrm{O}$ confined in MCM-41/C10 (pore size $2.8 \mathrm{~nm}$, black line) and in MCM-41/C18 matrix (pore size $4.5 \mathrm{~nm}$, red line). The diffraction patterns have been arbitrarily shifted along the vertical axis in order to have the same offset with respect to the origin of the reference frame.

served in the larger pores of MCM-41/C18 shows a slightly different diffraction signature, which is indeed more similar to the one observed for stacking faults ice $I$ obtained on warming up fast quenched amorphous salty solutions above their glass transition temperature ${ }^{51}$. This observation suggests that the effect on water structure imposed by the template (namely, the pore surface) depends on pore size, being larger for the smaller pores of MCM-41/C10. In turn, this would imply that a uniform ice structure, starting from a disordered template, can be found only after a number of stacked disordered ice layers is present in the pore volume, in agreement with recent simulation studies ${ }^{54}$.

At this point, there is strong evidence that confined $\mathrm{D}_{2} \mathrm{O}$ undergoes a crystallization transition around $240 \mathrm{~K}$, and, consequently, the maximum of the thermal expansion coefficient appearing at $240 \mathrm{~K}$ (Fig. 3B) is not related to the crossing of the Widom line but rather to the onset of a crystallization event. Based on the uncertainties associated with the measurement of the pore size ${ }^{39}$, being sensibly dependent on the particular method adopted to determine it, one may argue that a pore diameter of $2.8 \mathrm{~nm}$, as in MCM-41/C10, is too close to the reported value of $3 \mathrm{~nm}$ representing the largest size below which no water freezing can indeed be observed by DSC ${ }^{39}$. In addition, it has been shown that the freezing temperature of water can be lowered by about $60 \mathrm{~K}$ when confined in MCM-41 with pore diameter $\sim 3 \mathrm{~nm}^{40}$, a freezing point depression clearly not consistent with our observations.

All these issues can be rationalized as follows. The confinement-induced shift of the melting temperature, $\Delta T_{p}=$ $T_{0}-T_{p}$, where $T_{0}$ is the freezing temperature of bulk water and $T_{p}$ the freezing temperature of water confined, in a series of MCM41 material, was studied using DSC ${ }^{40}$. It was found that $\Delta T_{p}$ can be related to the pore radius $R$ by a modified form of the GibbsThomson equation:

$$
\Delta T_{p}=\frac{C_{G T}}{R-\lambda}
$$

where $C_{G T}$ is a constant value ${ }^{40}$, and the parameter $\lambda$ takes into account the presence of a layer of non-freezable water at the pore walls. In the Supplementary Information the procedure to obtain the thickness of $\lambda$ based on our diffraction data is described in detail.

We have estimated $\lambda=R-\left(C_{G T} / \Delta T_{p}\right)$ : for $R=1.4 \mathrm{~nm}$, we obtain $\lambda=0.38 \mathrm{~nm}$, that is very reasonable and in agreement with literature data for this sort of porous matrix, implying between 1 and 2 mono-layers of non-freezable water at the pore wall ${ }^{39,53}$. These results suggest a coexistence between freezable water, in the center of the pore, and non-freezable water at the pore wall below $240 \mathrm{~K}$. A rough estimate of the fraction of non-freezable water, $\phi$, corresponding to a layer with thickness $\lambda=0.38 \mathrm{~nm}$ gives $\phi=1-(1-\lambda / R)^{2}=47 \%$.

This outcome has several consequences. First, we can safely assume that changes in shape and position of the $\mathrm{D}_{2} \mathrm{O}$ peak around $Q=1.7 \AA^{-1}$ can be interpreted as a freezing phase transition of a not negligible fraction of water confined in MCM-41/C10 matrix. This, in turn, implies that the density of water inside the pores cannot be considered as constant across the pore, and therefore, the approach based on the Bragg peak intensity to determine the homogeneous density, $\rho_{m}$, of confined water has no ground. This observation rules out the existence of a minimum density of confined water as determined by SANS technique. The temperature dependence of $\rho_{m}$ reported in Fig. 3A should be then interpreted as resulting from a temperature-dependent rearrangement of a constant number of water molecules in the available pore volume, possibly due to increasing hydrogen-bonding upon cooling.

\section{Conclusions}

In conclusion, we have found that a non negligible fraction of water confined in MCM-41/C10 (pore diameter $2.8 \mathrm{~nm}$ ) crystallizes at $240 \mathrm{~K}$ as a stacking faults ice $I$ at the center of the pore. This fraction coexists with about one or two layers of non-freezable water at the pore wall. As a result, variation of water density across the pore cannot be considered as unchanging with temperature, and previous work, ${ }^{24-26,41}$, which appears to be based on this assumption, needs to be revisited. This conclusion neither implies that a density minimum in supercooled water does not exist, nor that there is no liquid-liquid transition to explain the water anomalies, but rather demonstrates that the claim of a density minimum in confined liquid water at $210 \mathrm{~K}$, as determined looking only at the temperature dependence of the intensity of the (10) Bragg peak of the 2D hexagonal internal structure of the porous matrix, is incorrect. Moreover, extension of the investigated $Q$ region both to lower and to higher $Q$ values, allows us on the one hand to rule out the presence of a maximum in density fluctuations, expected if a Widom line were being crossed in confined water, and, on the other hand, to identify a crystallization or other solidification event starting at about $240 \mathrm{~K}$. 


\subsection{Acknowledgments}

This work was supported within the CNR-CCLRC Agreement No. 01/9001 concerning collaboration in scientific research at the spallation neutron source ISIS. The financial support of the Consiglio Nazionale delle Ricerche and of the ANR Blanc International program PACS under reference no. ANR-13-IS04-000601 in this research is hereby acknowledged.

\section{References}

1 Angell C A, Kanno H (1976) Density maxima in high-pressure supercooled water and liquid silicon dioxide. Science 193: 1121 - 1122.

2 Angell C A, Bressel R D, Hemmati M, Sare E J, Tucker J C (2000) Water and its anomalies in perspective: tetrahedral liquids with and without liquid?liquid phase transitions. Phys. Chem. Chem. Phys. 2: 1559?1566.

3 Poole P H, Saika-Voivod I, Sciortino F (2005) Density minimum and liquid-liquid phase transition. J. Phys.: Condens. Matter 17: L431-L437.

4 Paschek D (2005) How the Liquid-Liquid Transition Affects Hydrophobic Hydration in Deeply Supercooled Water. Phys. Rev. Lett. 94: 217802-6.

5 Xu L M, Kumar P, Buldyrev S V, Chen S H, Poole P H, Sciortino F, Stanley H E (2005) Relation between the Widom line and the dynamic crossover in systems with a liquid-liquid phase transition. Proc. Natl. Acad. Sci. U. S. A. 102: 16558?16562.

6 Poole P H, Sciortino F, Essmann U, Stanley H E (1992) Phase beahviour ofmetastable water. Nature 360: 324-328.

7 Stanley H E, Cruz L, Harrington S T, Poole P H, Sastry S, Sciortino F, Starr F W, Zhang R (1997) Cooperative molecular motions in water: The liquid-liquid critical point hypothesis. Physica A 236: 19-37.

8 Buldyrev S V, Stanley H E (2003) A system with multiple liquid-liquid critical points. Physica A 330: 124 - 129.

9 Abascal L F, Vega C (2010) Widom line and the liquid-liquid critical point for the TIP4P/2005 water model. J. Chem. Phys. 133: 234502.

10 Gallo P, Amann-Winkel K, Angell C A, Anisimov M A, Caupin F, Chakravarty C, Lascaris E, Loerting T, Panagiotopoulos A Z, Russo J, Sellberg J A, Stanley H E, Tanaka H, Vega C, Xu L, Pettersson L G M (2016) Water: A Tale of Two Liquids. Chem. Rev. 116: 7463?7500.

11 Franzese G, Stanley H E (2002) Liquid-liquid critical point in a Hamiltonian model for water: analytic solution. J. Phys.: Condens. Matter 14: 2201.

12 Holten V, Kalová J, Anisimov M A, Sengers J V (2012) Thermodynamics of Liquid?Liquid Criticality in Supercooled Water in a Mean-Field Approximation. Int. J. Thermophys. 33: 758773.

13 Moore E B, Molinero V (2009) Growing correlation length in supercooled water. J. Chem. Phys. 130: 244505.

14 Moore E B, Molinero V (2011) Structural Transformation in Supercooled Water Controls the Crystallization Rate of Ice. Nature 479: 506-508.

15 Franzese G, Stanley H E (2007) The Widom line of super- cooled water. J. Phys.: Condens. Matter 19: 205126.

16 Sellberg J A, et al. (2014) Ultrafast X-ray probing of water structure below the homogeneous ice nucleation temperature. Nature 510: 381-384

17 Kim K H, SpâĂřh A, Pathak H, Perakis F, Mariedahl D, AmannWinkel K, Sellberg J A, Lee J H, Kim S, Park J, Nam K H, Katayama T, Nilsson A (2017) Maxima in the thermodynamic response and correlation functions of deeply supercooled water. Science 358: 1589-1593.

18 Caupin F, Holten V, Qiu C, Guillerm E, Wilke M, Frenz M, Teixeira J, Soper A K (2018) Comment on "Maxima in the thermodynamic response and correlation functions of deeply supercooled water". Science. 360: eaat1634.

19 Kittaka S, Ishimaru S, Kuranishi M, Matsuda T, Yamaguchi T (2006) Enthalpy and interfacial free energy changes of water capillary condensed in mesoporous silica, MCM-41 and SBA15. Phys. Chem. Chem. Phys. 8: 3223?3231.

20 Oguni M, Kanke Y, Nagoe A, Namba S (2011) Calorimetric Study of Water's Glass Transition in Nanoscale Confinement, Suggesting a Value of $210 \mathrm{~K}$ for Bulk Water. J. Phys. Chem. B 115: 14023 ? 14029.

21 Ricci M A, Bruni F, Giuliani A (2009) "Similarities" between confined and supercooled water. Faraday Discuss. 141: 347358.

22 Bruni F, Mancinelli R, Ricci M A (2012) How safe is to safely enter in the water no-man's land? J. Mol. Liq. 176: 39?43.

23 Soper A K (2011) Density minimum in supercooled confined water. Proc. Natl. Acad. Sci. U.S.A. 108: E1192.

24 Liu D, Zhang Y, Chen C-C, Mou C-Y, Poole P H, Chen S-H (2007) Observation of the density minimum in deeply supercooled confined water. Proc. Natl. Acad. Sci. U.S.A 104: 9570 $-9574$

25 Zhang Y, Faraone A, Kamitakahara W A, Liu K-H, Mou CY, Leao J B, Chang S, Chen S H (2011) Density hysteresis of heavy water confined in a nanoporous silica matrix. Proc. Natl. Acad. Sci. U.S.A 108:12206-12211.

26 Kamitakahara W A, Faraone A, Liu K-H, Mou C-Y (2012) Temperature dependence of structure and density for $\mathrm{D}_{2} \mathrm{O}$ confined in MCM-41-S. J. Phys.: Condens. Matter 24: 064106.

27 Liu K-H, Zhang Y, Lee J-J, Chen-C, Yeh Y-Q, Chen S-H, Mou. C$Y$ (2013) Density and anomalous thermal expansion of deeply cooled water confined in mesoporous silica investigated by synchrotron X-ray diffraction. J. Chem. Phys. 139: 064502.

28 Bertrand C E, Zhang Y, Chen S-H (2013) Deeply-cooled water under strong confinement: neutron scattering investigations and the liquid?liquid critical point hypothesis. Phys. Chem. Chem. Phys. 15: 721?745.

29 Mancinelli R, Bruni F, Ricci MA (2010) Controversial Evidence on the Point of Minimum Density in Deeply Supercooled Confined Water. J Phys. Chem. Lett. 1:1277-1282.

30 Gallo P, Rovere M, Chen S-H (2010) Dynamic Crossover in Supercooled Confined Water: Understanding Bulk Properties through Confinement. J. Phys. Chem. Lett. 1: 729-733.

31 Lerbret A, Lelong G, Mason P E, Saboungi M-L, Brady J W 
(2011) Water Confined in Cylindrical Pores: A Molecular Dynamics Study. Food Biophys. 6: 233?240.

32 Soper A K (2012) Density profile of water confined in cylindrical pores in MCM-41 silica. J. Phys.: Condens. Matter 24: 064107.

33 Erko M, Wallacher D, Hoell A, Hauss T, Zizak I, Paris O (2012) Density minimum of confined water at low temperatures: a combined study by small-angle scattering of X-rays and neutrons. Phys. Chem. Chem. Phys. 14: 3852.

34 Sattig M, Vogel M (2014) Dynamic Crossovers and Stepwise Solidification of Confined Water: A ${ }^{2} \mathrm{H}$ NMR Study. J. Phys. Chem. Lett. 5: 174-178.

35 Sattig M, Reutter S, Fujara F, Werner M, Buntkowsky G, Vogel M (2014) NMR studies on the temperature-dependent dynamics of confined water. Phys. Chem. Chem. Phys. 16: 1922919240.

36 Holten V, Qiu C, Guillerm E, Wilke M, Rička J, Frenz M, Caupin F (2017) Compressibility Anomalies in Stretched Water and Their Interplay with Density Anomalies. J. Phys. Chem Lett. 8: 5519?5522.

37 Bowron D T, Soper A K, Jones K, Ansell S, Birch S, Norris J, Perrott L, Riedel D, Rhodes N J, Wakefield S R, Botti A, Ricci M A, Grazzi F, Zoppi M (2010) NIMROD: The Near and InterMediate Range Order Diffractometer of the ISIS second target station. Rev. Sci. Instr. 81: 033905.

38 Lelong G, Bhattacharyya S, Kline S, Cacciaguerra T, Gonzalez MA, Saboungi, ML (2008) Effect of surfactant concentration on the Morphology and texture of MCM-41 materials. J. Phys. Chem. C 112: 10674-10680.

39 Jahnert S, Vaca ChÂůvez F, Schaumann G E, Schreiber A, SchËĘnhoff M, Findenegg G H (2008) Melting and freezing of water in cylindrical silica nanopores. Phys. Chem. Chem. Phys. 10: 6039.

40 Findenegg G H, Jahnert S, Akcakayiran D, Schreiber A (2008) Freezing and Melting of Water Confined in Silica Nanopores. ChemPhysChem. 9: 2651?2659.

41 Liu D, Zhang Y, Liu Y, Wu J, Chen C-C, Mou C-Y, Chen S-H (2008) Density measurement of 1-D confined water by small angle neutron scattering method: pore size and hydration level dependences. J. Phys. Chem. B 112: 4309 ? 4312

42 Liu K H, Zhang Y, Jeng U-S, Mou C-H (2015) Density of hy- drophobically confined deeply cooled water investigated by small angle X-ray scattering. J. Chem. Phys. 143: 094704.

43 Soper A K, Ricci M A (2000) Structures of High-Density and Low-Density Water. Phys. Rev. Lett. 84: 2881-2884.

44 Debenedetti P G (1998) Condensed matter: One substance, two liquids? Nature 392: 127?128.

45 Palmer J C, Martelli F, Liu Y, Car R, Panagiotopoulos A Z, Debenedetti P G (2014) Metastable liquid-liquid transition in a molecular model of water. Nature 510: 385 ?388.

46 Wang Z, Ito K, Leao J B, Harriger L, Liu Y, Chen S-H (2015) Liquid-Liquid Phase Transition and Its Phase Diagram in Deeply-Cooled Heavy Water Confined in a Nanoporous Silica Matrix. J. Phys. Chem. Lett. 6: 2009?2014.

47 Wang Z, Liu K-H, Harriger L, Leao J B, Chen S-H (2014) Evidence of the existence of the high-density and low-density phases in deeply-cooled confined heavy water under high pressures. J. Chem. Phys. 141: 014501

48 Hansen J-P, McDonald I R. Theory of Simple Liquids, Academic Press, Oxford, 2013.

49 Hansen T C, Koza M M, Kuhs W F (2008) Formation and annealing of cubic ice: I. Modelling of stacking faults. J. Phys.: Condens. Matter 20: 285104.

50 Malkin T L, Murray B J, Salzmann C, Molinero V, Pickering S J, Whale T F (2015) Stacking Disorder in Ice I. Phys. Chem. Chem. Phys. 17: 60-76.

51 Ludl A-A, Bove L E, Saitta A M, Salanne M, Hansen T C, Bull C L, Gaal R, Klotz S (2015) Structural characterization of eutectic aqueous $\mathrm{NaCl}$ solutions under variable temperature and pressure conditions. Phys. Chem. Chem. Phys. 17: 1405414063.

52 Morishige K, Kawano, K (1999) Freezing and melting of water in a single cylindrical pore: The pore-size dependence of freezing and melting behavior. J. Chem. Phys. 110: 48674872.

53 Schreiber A, Ketelsen I, Findenegg G H (2001) Melting and freezing of water in ordered mesoporous silica materials. Phys. Chem. Chem. Phys. 3: 1185-1190.

54 Leoni F, Franzese G (2016) Effects of confinement between attractive and repulsive walls on the thermodynamics of an anomalous fluid. Phys. Rev. E. 94: 062604. 